\title{
Global Genome Comparative Analysis Reveals Insights of Resistome and Life-Style Adaptation of Pseudomonas putida Strain T2-2 in Oral Cavity
}

\author{
Xin Yue Chan, Kah Ooi Chua, Kah Yan How, Wai-Fong Yin, and Kok-Gan Chan \\ Division of Genetics and Molecular Biology, Institute of Biological Sciences, Faculty of Science, University of Malaya, \\ 50603 Kuala Lumpur, Malaysia
}

Correspondence should be addressed to Kok-Gan Chan; kokgan@um.edu.my

Received 8 June 2014; Revised 8 September 2014; Accepted 14 September 2014; Published 10 November 2014

Academic Editor: Christopher S. Henry

Copyright (C) 2014 Xin Yue Chan et al. This is an open access article distributed under the Creative Commons Attribution License, which permits unrestricted use, distribution, and reproduction in any medium, provided the original work is properly cited.

\begin{abstract}
Most Pseudomonas putida strains are environmental microorganisms exhibiting a wide range of metabolic capability but certain strains have been reported as rare opportunistic pathogens and some emerged as multidrug resistant $P$. putida. This study aimed to assess the drug resistance profile of, via whole genome analysis, P. putida strain T2-2 isolated from oral cavity. At the same time, we also compared the nonenvironmental strain with environmentally isolated $P$. putida. In silico comparative genome analysis with available reference strains of $P$. putida shows that T2-2 has lesser gene counts on carbohydrate and aromatic compounds metabolisms, which suggested its little versatility. The detection of its edd gene also suggested T2-2's catabolism of glucose via ED pathway instead of EMP pathway. On the other hand, its drug resistance profile was observed via in silico gene prediction and most of the genes found were in agreement with drug-susceptibility testing in laboratory by automated VITEK 2 . In addition, the finding of putative genes of multidrug resistance efflux pump and ATP-binding cassette transporters in this strain suggests a multidrug resistant phenotype. In summary, it is believed that multiple metabolic characteristics and drug resistance in $P$. putida strain T2-2 helped in its survival in human oral cavity.
\end{abstract}

\section{Introduction}

Pseudomonas putida are aerobic Gram-negative, nonlactosefermenting, and oxidase-positive bacteria [1]. As a member of the fluorescent group of Pseudomonas, $P$. putida produces pyoverdin, a yellow-green pigment that fluoresces under UV light and is grouped as nonphytopathogenic, nonnecrogenic strains together with $P$. aeruginosa and $P$. fluorescens $[2,3]$. Like the other members in this genus, the species exhibits a diverse metabolic capability making it famous to be able to inhabit a wide range of environments including soil that is contaminated with aromatic and toxic compounds, freshwater, and surfaces of living organisms [4]. To date, $P$. putida has been described with ability to degrade nicotine [5], benzoate [6], phenol [7], toluene [8], and alkane [9].

In spite of the fact that it is ubiquitous in various environments, isolation of $P$. putida from clinical specimen is rare. However, cases of $P$. putida infections had been reported and occurrence of the species in catheter caused outbreak of nosocomial infections which contributed to bacteremia in adult patients [10] as well as neonates [11]. In addition, in a review on $P$. putida infections of marine military personnel, a majority of the infections reported were cases of bacteremia, urinary tract infections, and pneumonia as well as uncommon infections on skins and soft tissues, peritoneum, central nervous system, ears, joints, and cornea [3]. These findings are alarming as $P$. putida is becoming a threat to public health care. This is especially critical when a P. putida strain HB3267 had been reported as multidrug resistant recently, which resists beta-lactams antibiotics, fluoroquinolone, macrolides, aminoglycosides, polymyxin, and polyketide [12].

As a species commonly found in the environment, association of $P$. putida as commensal organism in human is rarely described. A strain of the species, $P$. putida T2-2, had been isolated from a healthy individual's tongue surface and reported for its quorum sensing activity [13]. As there is 
reason to believe that the species is an opportunistic pathogen with ability to cause life-threatening diseases in human, it raises our concern about the emergence of the multidrug resistant strains. Consequently, in order to assess the difference in genomic profile and antibiotic resistance of clinically isolated $P$. putida and those obtained from the environment, P. putida strain T2-2 (hereafter referred to as T2-2) that was previously identified as commensal organism on a healthy individual's tongue surface was employed as reference culture for a comparative genome analysis. In this experiment, we whole-genome sequenced this isolate using next generation sequencing and performed genome analysis on the whole genome sequences of T2-2. We then furthered our study and reported the findings from comparative analysis between the draft genome of T2-2 and available whole genome of several environmental P. putida strains in the database.

\section{Materials and Methods}

2.1. Isolation of P. putida Strain T2-2 and Genomic DNA Extraction. Isolation and characterization of bacteria strain T2-2 have been described by Chen et al., 2013 [13]. Strain T2-2 was maintained and cultured in LB medium. The genomic DNA of strain T2-2 was extracted and purified with QIAamp DNA minikit (Qiagen). The extracted DNA was examined by NanoDrop Spectrophotometer (Thermo Scientific) for quality and quantified by Qubit 2.0 fluorometer (Life Technologies) using dsDNA High Sensitivity Assay Kit (Invitrogen). Extracted genomic DNA was stored in $-20^{\circ} \mathrm{C}$.

2.2. Library Preparation and Whole Genome Sequencing. The sequencing library was prepared using TruSeq DNA Sample Preparation Kit (v2) (Illumina) and subjected to next generation sequencing (NGS) on HiSeq 2000 (Illumina) platform [14].

2.3. Reference Genome Assembly. Closely related P. putida strains were determined from dendrogram based on genomic BLAST from National Center for Biotechnology Information (NCBI) [15]. Complete genomes of closely related $P$. putida and RAST available strains were downloaded from NCBI as reference genomes. Reference genome assembly was performed with CLC Genomic Workbench (v6.0.5) [16].

2.4. Genome Assembly and Annotation. The raw reads generated were trimmed and de novo assembled with CLC Genomic Workbench (v6.0.5). Contigs with minimal length of $200 \mathrm{bp}$ and coverage of 30-fold were selected for subsequent analysis. The genes were annotated with RAST (rapid annotation using subsystem technology) (v4) [17]. The number of rRNA genes was determined with RNAmmer [18] while tRNA genes were predicted with RAST [17].

2.5. Comparative Genome Analysis. The draft genome of T22 was used as reference genome and compared with genome of $P$. putida GB-1 (hereafter referred to as GB-1), P. putida F1 (hereafter referred to as F1), and P. putida W619 (hereafter referred to as W619) available in the RAST database.
2.6. Antibiotic Susceptibility Testing. McFarland 0.5 standard in $0.45 \%$ sodium chloride $(\mathrm{NaCl})$ was used as standard in measuring the turbidity of cell suspension. Bacteria turbidity was adjusted to $0.5-0.63$ in $3 \mathrm{~mL}$ of $0.45 \% \mathrm{NaCl}$ (AirLife) and measured with densitometer (bioMerieux) [19]. AST-GN83 card was read by Vitek 2 (bioMerieux) and the antibiogram was generated by the software [20]. The antimicrobials tested were ampicillin, amoxicillin/clavulanic acid, ampicillin/sulbactam, piperacillin/tazobactam, cefazolin, cefuroxime, cefuroxime axetil, cefoxitin, cefotaxime, ceftazidime, ceftriaxone, cefepime, aztreonam, meropenem, amikacin, gentamicin, ciprofloxacin, nitrofurantoin, and trimethoprim/sulfamethoxazole. The result was analyzed with VITEK 2 Advanced Expert System.

2.7. Molecular Detection of 6-Phosphogluconate Dehydratase Gene (edd) by PCR. The edd gene was amplified using forward primer: $5^{\prime}$-ACCAGCCTTACTTGCACTTC- $3^{\prime}$ and reverse primer: $5^{\prime}$-CCATCACTTCCATCACCAACT- $3^{\prime}$. Pseudomonas putida S13 (hereafter referred to as S13) was employed as positive control. The PCR condition was as follows: denaturation at $95^{\circ} \mathrm{C}$ for $2.5 \mathrm{~min}$; denaturation at $95^{\circ} \mathrm{C}$ for $40 \mathrm{~s}$, annealing at $54.5^{\circ} \mathrm{C}$ for $40 \mathrm{~s}$, and extension at $72^{\circ} \mathrm{C}$ for $30 \mathrm{~s}$ repeated for $35 \mathrm{cycles}$. The final elongation step was at $72^{\circ} \mathrm{C}$ for $3 \mathrm{~min}$. PCR amplification was performed in $20 \mu \mathrm{L}$ volume with Bio-Rad T100 Thermal Cycler. The amplified DNA was purified and the gene sequence was confirmed by Sanger sequencing.

2.8. Gene Alignment and Comparison. Amino acid sequences of edd genes from P. putida T2-2 and S13 were generated based on their DNA sequences using MEGA (v5.2.2). Their DNA sequences were aligned with reference sequences from NCBI [21]. Dot plots of these aligned amino acids and DNA sequences were generated using LAST [22].

\section{Results and Discussion}

High quality reads from strain T2-2 were mapped against reference genome $P$. putida strains W619, HB3267, GB-1, and F1 and the details were listed in Table 1. The genome of $P$. putida strain T2-2 was mapped with reference genomes with at least $80 \mathrm{X}$ coverage (Table 1). However, based on the reference mapping result, less than $70 \%$ of the reads were mapped to the reference genomes. This suggested that genomic differences with probable insertions or deletions occurred in the genome of strain T2-2.

Owing to the low percentage of reads that were mapped to the reference genome, de novo assembly was performed to acquire more information of the genome of P. putida T2-2. The draft genome from the de novo assembly resulted in 389 contigs with a genome size of $5.5 \mathrm{Mbp}$. The $\mathrm{G}+\mathrm{C}$ content of the genome is $62.57 \%$ with an N50 of $16.3 \mathrm{~kb}$ and a total of 5,053 coding DNA sequences (CDSs) were predicted by RAST, 4 copies of rRNA operons as predicted by RNAmmer and 74 copies of tRNA genes as predicted by RAST. The draft genome is deposited into DDBJ/EMBL/GenBank under the 
TABle 1: Summary of reference genome mapping of $P$. putida T2-2 against the complete genome of $P$. putida W619, $P$. putida HB3267, $P$. putida F1, and P. putida GB-1.

\begin{tabular}{lccccc}
\hline Reference genome & $\begin{array}{c}\text { GenBank accession } \\
\text { number }\end{array}$ & $\begin{array}{c}\text { Consensus length, } \\
(\mathrm{Mbp})\end{array}$ & $\begin{array}{c}\text { Average } \\
\text { coverage }\end{array}$ & $\begin{array}{c}\text { Reads map to reference } \\
\text { genome, \% }\end{array}$ & $\begin{array}{c}\text { Bases map to } \\
\text { reference genome, \% }\end{array}$ \\
\hline P. putida W619 & CP000949.1 & 5.77 & 84 & 49.51 & 50.19 \\
P. putida HB3267 & CP003738.1 & 5.88 & 158 & 61.55 & 62.11 \\
P. putida F1 & CP000712.1 & 5.96 & 94 & 61.51 & 62.14 \\
P. putida GB-1 & CP000926.1 & 6.08 & 142 & 62.39 & 62.99 \\
\hline
\end{tabular}

TABLE 2: Summary of T2-2 draft genome compared with other $P$. putida complete genomes available in RAST.

\begin{tabular}{lccc}
\hline $\begin{array}{l}\text { Sample of } P . \\
\text { putida }\end{array}$ & $\begin{array}{c}\text { Genome ID in } \\
\text { RAST }\end{array}$ & $\begin{array}{c}\text { Genome size } \\
(\mathrm{Mbp})\end{array}$ & Total CDSs \\
\hline T2-2 & 303.65 & 5.5 & 5053 \\
F1 & 351746.4 & 6.0 & 5252 \\
GB-1 & 76869.3 & 6.1 & 5410 \\
W619 & 390235.3 & 5.8 & 5182 \\
\hline
\end{tabular}

accession JALX00000000. The version described in this paper is the first version, JALX01000000.

From RAST annotation, the closest neighbours of T2-2 were P. putida B6-2 (score 545), followed by P. putida F1 (score 502) and P. putida KT2440 and P. putida GB-1 (score 494). The present whole genome analysis of bacterial identification was in agreement with the previous report that relied on phylogenetics analysis of its $16 \mathrm{~S}$ rDNA (Accession number: HQ907954) which clearly identified T2-2 as P. putida [13]. The 5,053 CDSs predicted by RAST were divided into subsystems and shown in Figure 1. All essential genes identified in T2-2 were present in the genome of T2-2 and the genes functions were assigned to nutrients metabolism, respiration, and cell division.

The summary of T2-2 draft genome and other selected genomes of environmental $P$. putida strains is shown in Table 2. Comparison on their subsystem distributions revealed the nonenvironmental lifestyle of T2-2 with lesser gene count (336) on carbohydrates metabolism as compared to GB-1 (445), F1 (473), and W619 (410). With regard to one-carbon $(\mathrm{Cl})$ compounds metabolism, no CDS for serineglyoxylate cycle that was responsible for $\mathrm{Cl}$-compounds metabolism was detected by RAST annotation in T2-2. Similar finding was obtained for W619 that was isolated from rhizosphere of plants as endophyte [23]. It is believed that serine-glyoxylate cycle enables GB-1 and F1 to assimilate C1 compounds in the environment such as rhizoplane [24].

Besides, the glucose metabolism in P. putida T2-2 was studied and its ability to consume glucose was observed (data not shown). Due to the absence of 6-phosphofructokinase for Embden-Meyerhof-Parnas (EMP) route, glucose metabolism in P. putida is mainly through Entner-Doudoroff (ED) pathway [25]. In this study, the PCR-amplified 471 bp DNA fragment was predicted to be the edd gene that codes for 6-phosphogluconate dehydratase (Figure 2(a)) in T2-2. Similar PCR product was obtained from the same species
P. putida strain S13 (Figures 2(a) and 2(b)). Based on the gene alignment and comparison from LAST, it showed high similarity with 6-phosphogluconate dehydratase of the reference genome Pseudomonas sp. BAY1663 (Accession number: gi588313542) (Figure 2(c)). In both ED and EMP pathways, the hexose sugars are phosphorylated and subsequently cleaved by aldolase enzymes into two three-carbon products [26]. However, ED pathway was distinct from EM pathway by two key enzymes which were the 6-phosphogluconate dehydratase (Edd) and KDPG aldolase (Eda) [26].

Analysis on CDS under the subsystem of virulence, disease, and defence in $P$. putida strain T2-2 revealed the presence of genes responsible for iron acquisition which is an important aspect of virulence due to the scarcity of iron available for bacteria in the human body [27]. Besides, CDS encoding for resistance and tolerance to heavy metals including copper, arsenic, chromium, and cobalt-zinc-cadmium were detected from strain T2-2 as well as W619, HB3267, F1, and GB-1.

In addition, CDSs responsible for lysozyme inhibition, beta-lactamases, and resistance towards antibiotic fluoroquinolones are present in their genomes as well. This prediction in T2-2 was supported by antibiogram with higher than expected minimal inhibitory concentrations (MICs) readings in strain T2-2 when tested with $\beta$-lactam antibiotics including ampicillin, amoxicillin, sulbactam, cefazolin, cefuroxime, cefuroxime axetil, cefoxitin, and fluoroquinolones compound, nitrofurantoin (Table 3). Even though P. putida infection in human rarely happens, we should be aware of the emergence of multidrug resistant strains as it had been reported as the causal agent of nosocomial infection, urinary tract infection, and other diseases $[3,28,29]$.

However, a clear, distinctive characteristic of the T22 strain is its possession of a group of CDSs encoding for resistance-nodulation-division (RND) multidrug efflux pumps that are not present in the genomes of the other three P. putida strains selected from RAST (see Supplementary Table 1 in Supplementary Material available online at http://dx.doi.org/10.1155/2014/930727). These RND pumps function as a tripartite protein complex that consists of the outer membrane protein, membrane fusion protein (the periplasmic component), and inner membrane component [30]. The complex forms a channel across the cell membrane, enabling the pumping of molecules (such as antibiotic) across the bacterial periplasm and the outer membrane into the external medium efficiently as this process is driven by energy derived from proton gradient [30]. In Pseudomonas 

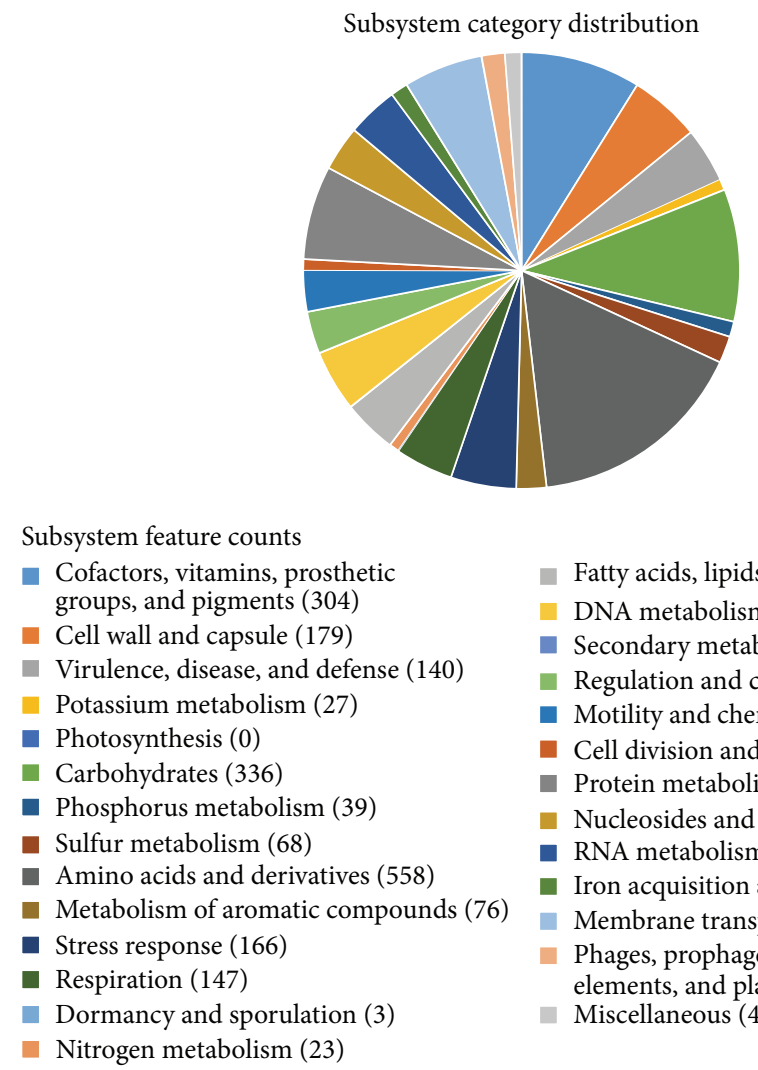

Fatty acids, lipids, and isoprenoids (137)

DNA metabolism (156)

- Secondary metabolism (0)

- Regulation and cell signaling (107)

- Motility and chemotaxis (106)

- Cell division and cell cycle (27)

- Protein metabolism (238)

- Nucleosides and nucleotides (114)

- RNA metabolism (130)

- Iron acquisition and metabolism (44)

- Membrane transport (201)

- Phages, prophages, transposable elements, and plasmids (59)

- Miscellaneous (42)

FIGURE 1: Subsystem distribution of $P$. putida T2-2 genome. Whole genome sequence was annotated and the subsystem distribution was generated from RAST.

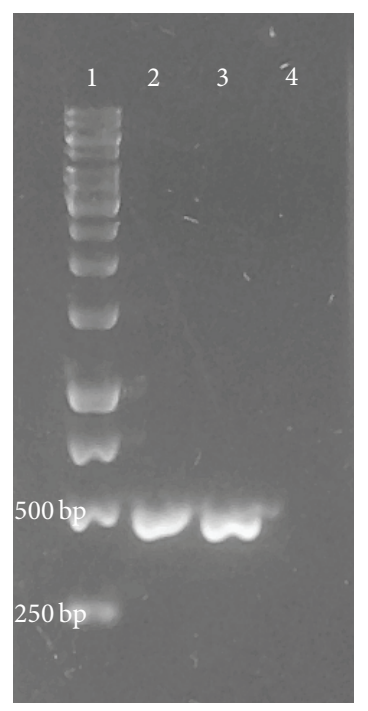

(a)

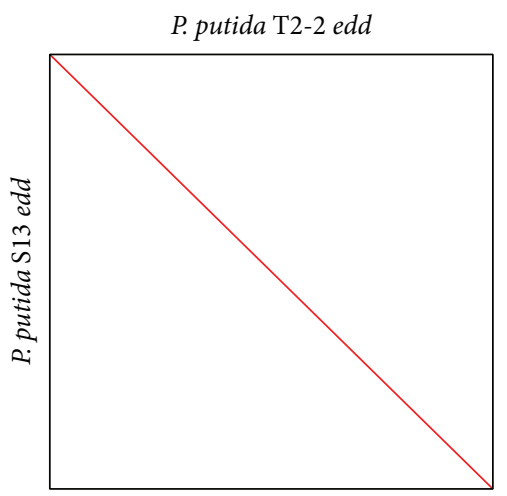

(b)

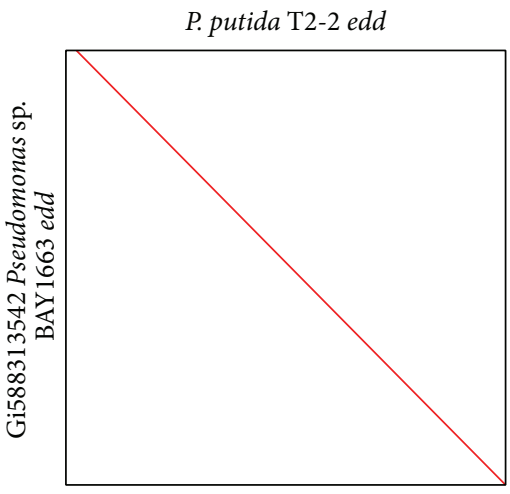

(c)

FIgure 2: (a) Agarose gel image for 1: $1 \mathrm{~kb}$ DNA ladder; 2: PCR product edd gene in P. putida T2-2; 3: PCR product edd gene in P. putida S13 which serves as positive control, and 4: negative control. (b) Dot plot from DNA sequence of edd gene of $P$. putida T2-2 against $P$. putida S13. (c) Dot plot from amino acid sequence of edd gene of $P$. putida T2-2 against reference gene Pseudomonas strain BAY1663. 
TABle 3: Antibiogram of Pseudomonas putida strain T2-2. Antimicrobial compounds with MICs higher than expected were regarded as resistant by strain T2-2 and marked as " $\mathrm{R}$ " while the compounds with lower or equal to expected MICs were interpreted as sensitive for strain T2-2 and were marked as "S".

BioMerieux customer:

University of Malaya

Laboratory report

System number:

Isolate group: $\mathrm{t} 2$ 2-1

Card type: AST-GN83 Testing Instrument: 000015F14C94 (Serial No. 4058)

Organism quantity:

Selected organism: Pseudomonas spp.

Comments:

Identification information

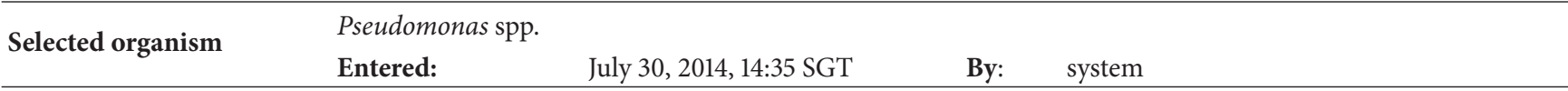

Analysis messages:

The following antibiotic(s) are suppressed from analysis:

aztreonam

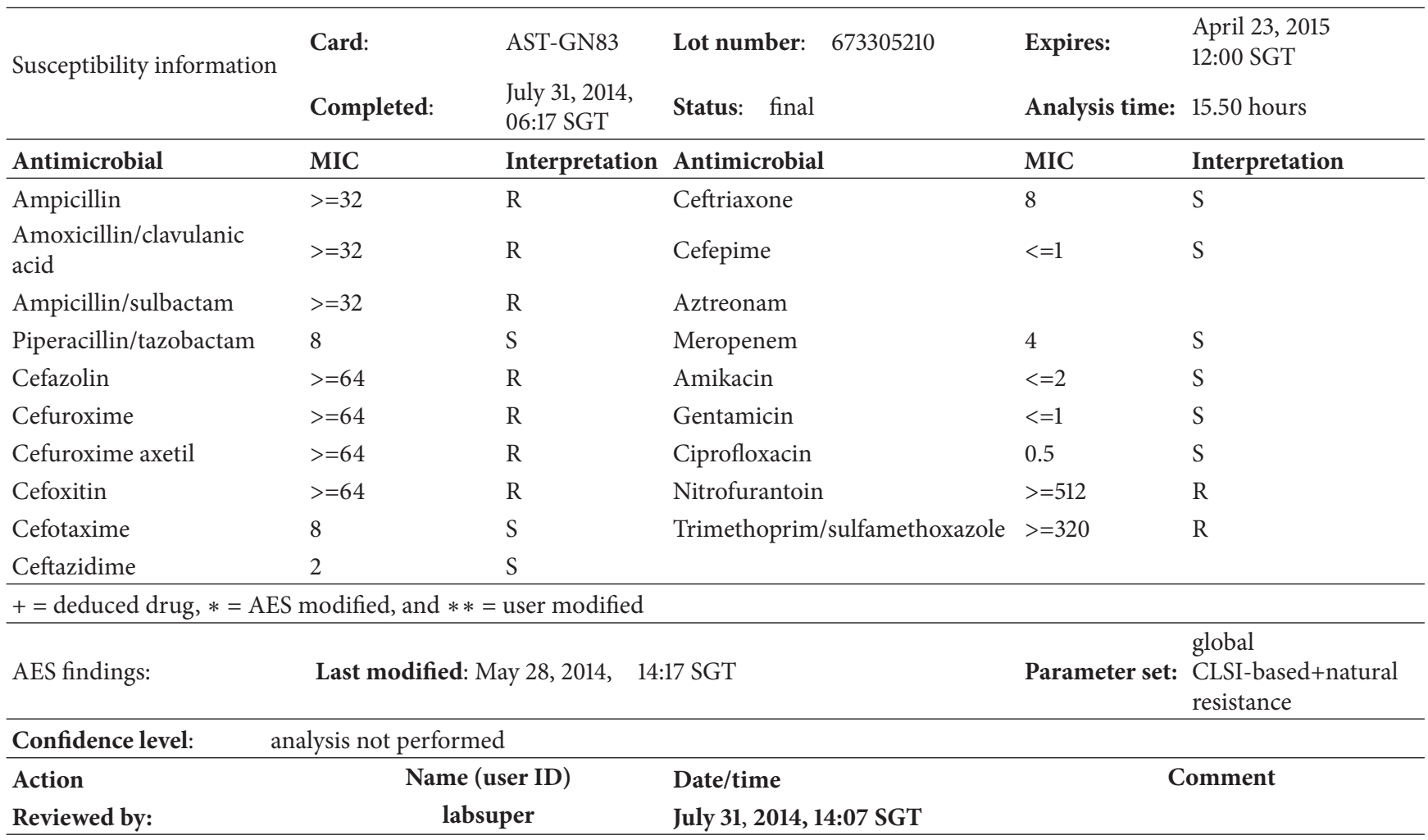

Installed VITEK 2 systems version: 07.01.

MIC interpretation guideline: global CLSI-based. Therapeutic interpretation guideline: natural resistance.

AES parameter set name: global CLSI-based+natural resistance. $\quad$ AES parameter last modified: May 28, 2014, 14:17 SGT.

aeruginosa, the RND pumps elevate its drug resistance by causing a slower accumulation of drug inside the cell [31].

The schematic diagram of RND genes organization encoding the mentioned system in T2-2 is shown in Figure 3. According to RAST annotation, the CDSs encode proteins including membrane fusion protein CmeA, inner membrane transporter $\mathrm{CmeB}$, and outer membrane lipoprotein $\mathrm{CmeC}$ (Figure 3). The proteins CmeABC have been reported as multidrug efflux system in Campylobacter jejuni contributing to resistance towards a wide range of antibiotics, heavy metals, bile salt, and other antimicrobial agents [32]. Interestingly, the presence of the system as a complete tripartite protein complex is revealed by draft genome analysis in T2-2 supporting resistance of this strain towards the therapeutic antimicrobial agents [30]. 


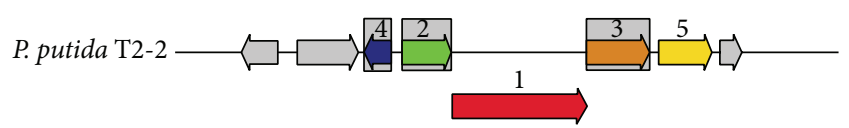

FIGURE 3: Genes organization of multidrug resistance efflux pumps identified by RAST annotation of T2-2 genome. (1) RND efflux system, inner membrane transporter, $\mathrm{CmeB}$; (2) membrane fusion protein of RND family multidrug efflux pump; (3) RND efflux system, outer membrane lipoprotein, CmeC; (4) transcription repressor of multidrug efflux pump acrAB operon, TetR(AcR) family.

Besides, a wide range of adenosine triphosphate (ATP) binding cassette $(\mathrm{ABC})$ transporters has been discovered in genome of T2-2, which are not noticed in the other three reference genomes of environmental strains. These include the $\mathrm{ABC}$ transporters for alkyl phosphate, oligopeptide, branched amino acids, and dipeptide. These ABC transporters play an important role in the active transport systems of the archaea, eubacteria and eukaryotic cells $[33,34]$. They are responsible for importing and exporting wide variety of substrates in and out of the bacteria cell, which include carbohydrates and sugars, amino acids, and peptides, as well as metal ions [35]. The presence of the $\mathrm{ABC}$ transporters in T2-2 is believed to assist this bacterium in uptake of partially digested solutes in the environment of oral cavity, making it adapt better in the habitat.

However, coupling of $\mathrm{ABC}$ transporters and multidrug efflux pump encourages the emergence of multidrug resistant strain as the energy (ATP) supplied by ABC transporter enables the active translocation of antibiotic molecules [36]. This might make T2-2 able to survive or even countermeasure the antibacterial drugs it encountered in colonization of the oral cavity.

\section{Conclusions}

The comparative analysis of draft genome of P. putida T2-2 revealed lesser gene counts in metabolism of carbohydrates and aromatic compounds as compared to selected environmental strains. In order to adapt to the human oral cavity, this strain possesses extra putative $\mathrm{ABC}$ transporter systems for various substrates which might help in its survival in the oral cavity. Moreover, the putative presence of additional multidrug efflux pump genes in the genome reveals its possible multidrug resistant nature.

\section{Conflict of Interests}

The authors declare that there is no conflict of interests regarding the publication of this paper.

\section{Acknowledgments}

Kok-Gan Chan thanks the University of Malaya High Impact Research (HIR) Grant (UM-MOHE HIR Grant UM.C/ 625/1/HIR/MOHE/CHAN/14/1, no. H-50001-A000027). The authors thank S. Chin and K. Purmal for the oral bacteria isolation work.

\section{References}

[1] C. Aumeran, C. Paillard, F. Robin et al., "Pseudomonas aeruginosa and Pseudomonas putida outbreak associated with contaminated water outlets in an oncohaematology paediatric unit," Journal of Hospital Infection, vol. 65, no. 1, pp. 47-53, 2007.

[2] G. R. Botelho and L. C. Mendonça-Hagler, "Fluorescent Pseudomonads associated with the rhizosphere of crops: an overview," Brazilian Journal of Microbiology, vol. 37, no. 4, pp. 401-416, 2006.

[3] R. J. Carpenter, J. D. Hartzell, J. A. Forsberg, B. S. Babel, and A. Ganesan, "Pseudomonas putida war wound infection in a US Marine: a case report and review of the literature," Journal of Infection, vol. 56, no. 4, pp. 234-240, 2008.

[4] M. W. Silby, C. Winstanley, S. A. C. Godfrey, S. B. Levy, and R. W. Jackson, "Pseudomonas genomes: diverse and adaptable," FEMS Microbiology Reviews, vol. 35, no. 4, pp. 652-680, 2011.

[5] H. Tang, Y. Yao, L. Wang et al., "Genomic analysis of Pseudomonas putida: genes in a genome island are crucial for nicotine degradation," Scientific Reports, vol. 2, article 377, 2012.

[6] K.-C. Loh and S.-S. Chua, "Ortho pathway of benzoate degradation in Pseudomonas putida: induction of meta pathway at high substrate concentrations," Enzyme and Microbial Technology, vol. 30, no. 5, pp. 620-626, 2002.

[7] Á. A. M. G. Monteiro, R. A. R. Boaventura, and A. E. Rodrigues, "Phenol biodegradation by Pseudomonas putida DSM 548 in a batch reactor," Biochemical Engineering Journal, vol. 6, no. 1, pp. 45-49, 2000.

[8] T. Krell, J. Lacal, M. E. Guazzaroni et al., "Responses of Pseudomonas putida to toxic aromatic carbon sources," Journal of Biotechnology, vol. 160, no. 1-2, pp. 25-32, 2012.

[9] J. B. van Beilen, S. Panke, S. Lucchini, A. G. Franchini, M. Röthlisberger, and B. Witholt, "Analysis of Pseudomonas putida alkane-degradation gene clusters and flanking insertion sequences: evolution and regulation of the alk genes," Microbiology, vol. 147, no. 6, pp. 1621-1630, 2001.

[10] Y. Yoshino, T. Kitazawa, M. Kamimura, K. Tatsuno, Y. Ota, and H. Yotsuyanagi, "Pseudomonas putida bacteremia in adult patients: five case reports and a review of the literature," Journal of Infection and Chemotherapy, vol. 17, no. 2, pp. 278-282, 2011.

[11] O. Boullègue, R. Mzoughi, F.-X. Weill et al., "Outbreak of Pseudomonas putida bacteraemia in neonatal intensive care unit," Journal of Hospital Infection, vol. 57, no. 1, pp. 88-91, 2004.

[12] L. Molina, Z. Udaondo, E. Duque et al., "Antibiotic resistance determinants in a Pseudomonas putida strain isolated from a hospital," PLoS ONE, vol. 9, no. 1, Article ID e81604, 2014.

[13] J.-W. Chen, S. Chin, K. K. Tee, W.-F. Yin, Y. M. Choo, and K.G. Chan, " $N$-acyl homoserine lactone-producing Pseudomonas putida strain T2-2 from human tongue surface," Sensors, vol. 13, no. 10, pp. 13192-13203, 2013.

[14] K.-G. Chan, C. S. Wong, W. F. Yin, and X. Y. Chan, "Draft genome sequence of quorum-sensing and quorum-quenching Pseudomonas aeruginosa strain MW3a," Genome Announcements, vol. 2, no. 2, Article ID e00258-14, 2014.

[15] A. Acland, R. Agarwala, T. Barrett et al., "Database resources of the national center for biotechnology information," Nucleic Acids Research, vol. 41, pp. D8-D20, 2013. 
[16] X. Y. Chan, K. H. Chua, S. D. Puthucheary, W.-F. Yin, and K.-G. Chan, "Draft genome sequence of an Aeromonas sp. Strain 159 clinical isolate that shows quorum-sensing activity," Journal of Bacteriology, vol. 194, no. 22, p. 6350, 2012.

[17] R. K. Aziz, R. Agarwala, T. Barrett et al., "The RAST Server: rapid annotations using subsystems technology," BMC Genomics, vol. 9, p. 75, 2008.

[18] K. Lagesen, P. Hallin, E. A. Rødland, H.-H. Stærfeldt, T. Rognes, and D. W. Ussery, "RNAmmer: consistent and rapid annotation of ribosomal RNA genes," Nucleic Acids Research, vol. 35, no. 9, pp. 3100-3108, 2007.

[19] T. K. W. Ling, P. C. Tam, Z. K. Liu, and A. F. Cheng, "Evaluation of VITEK 2 rapid identification and susceptibility testing system against gram-negative clinical isolates," Journal of Clinical Microbiology, vol. 39, no. 8, pp. 2964-2966, 2001.

[20] M. J. Bruins, P. Bloembergen, G. J. Ruijs, and M. J. Wolfhagen, "Identification and susceptibility testing of Enterobacteriaceae and Pseudomonas aeruginosa by direct inoculation from positive BACTEC blood culture bottles into Vitek 2," Journal of Clinical Microbiology, vol. 42, no. 1, pp. 7-11, 2004.

[21] K. Tamura, G. Stecher, D. Peterson, A. Filipski, and S. Kumar, "MEGA6: molecular evolutionary genetics analysis version 6.0," Molecular Biology and Evolution, vol. 30, no. 12, pp. 2725-2729, 2013.

[22] X. Y. Chan, C. Y. Chang, K. W. Hong, K. K. Tee, W. F. Yin, and K. G. Chan, "Insights of biosurfactant producing Serratia marcescens strain W2.3 isolated from diseased tilapia fish: a draft genome analysis," Gut Pathogens, vol. 5, no. 1, article 29, 2013.

[23] X. Wu, S. Monchy, S. Taghavi, W. Zhu, J. Ramos, and D. van der Lelie, "Comparative genomics and functional analysis of nichespecific adaptation in Pseudomonas putida," FEMS Microbiology Reviews, vol. 35, no. 2, pp. 299-323, 2011.

[24] N. Korotkova, L. Chistoserdova, V. Kuksa, and M. E. Lidstrom, "Glyoxylate regeneration pathway in the methylotroph Methylobacterium extorquens AM1," Journal of Bacteriology, vol. 184, no. 6, pp. 1750-1758, 2002.

[25] M. Chavarría, P. I. Nikel, D. Pérez-Pantoja, and V. De Lorenzo, "The Entner-Doudoroff pathway empowers Pseudomonas putida KT2440 with a high tolerance to oxidative stress," Environmental Microbiology, vol. 15, no. 6, pp. 1772-1785, 2013.

[26] N. Peekhaus and T. Conway, "What's for dinner?: EntnerDoudoroff metabolism in Escherichia coli," Journal of Bacteriology, vol. 180, no. 14, pp. 3495-3502, 1998.

[27] K.-G. Chan, "Exochelin production in Mycobacterium neoaurum," International Journal of Molecular Sciences, vol. 10, no. 1, pp. 345-353, 2009.

[28] G. Lombardi, F. Luzzaro, J.-D. Docquier et al., "Nosocomial infections caused by multidrug-resistant isolates of Pseudomonas putida producing VIM-1 metallo- $\beta$-lactamase," Journal of Clinical Microbiology, vol. 40, no. 11, pp. 4051-4055, 2002.

[29] J.-D. Docquier, M. L. Riccio, C. Mugnaioli et al., "IMP-12, a new plasmid-encoded metallo- $\beta$-lactamase from a Pseudomonas putida clinical isolate," Antimicrobial Agents and Chemotherapy, vol. 47, no. 5, pp. 1522-1528, 2003.

[30] D. M. Fernando and A. Kumar, "Resistance-nodulationdivision multidrug efflux pumps in gram-negative bacteria: role in virulence," Antibiotics, vol. 2, pp. 163-181, 2013.

[31] H. Nikaido, "Multidrug efflux pumps of gram-negative bacteria," Journal of Bacteriology, vol. 178, no. 20, pp. 5853-5859, 1996.
[32] J. Lin, L. O. Michel, and Q. Zhang, "CmeABC functions as a multidrug efflux system in Campylobacter jejuni," Antimicrobial Agents and Chemotherapy, vol. 46, no. 7, pp. 2124-2131, 2002.

[33] C. F. Higgins, "ABC transporters: physiology, structure and mechanism-an overview," Research in Microbiology, vol. 152, no. 3-4, pp. 205-210, 2001.

[34] K. Tomii and M. Kanehisa, "A comparative analysis of $\mathrm{ABC}$ transporters in complete microbial genomes," Genome Research, vol. 8, no. 10, pp. 1048-1059, 1998.

[35] H. S. Garmory and R. W. Titball, "ATP-binding cassette transporters are targets for the development of antibacterial vaccines and therapies," Infection and Immunity, vol. 72, no. 12, pp. 6757$6763,2004$.

[36] G. Chang, "Multidrug resistance ABC transporters," FEBS Letters, vol. 555, no. 1, pp. 102-105, 2003. 

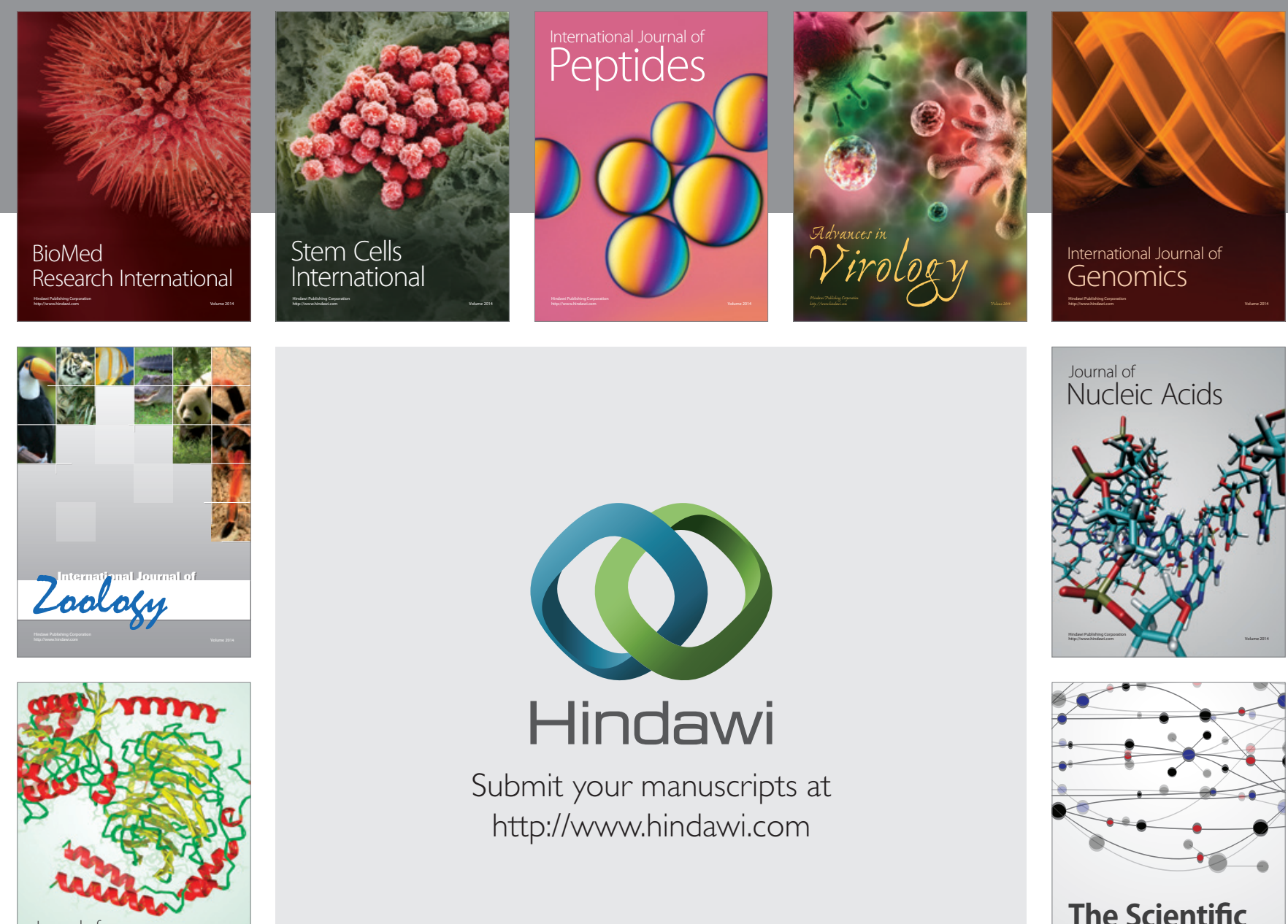

Submit your manuscripts at

http://www.hindawi.com

Journal of
Signal Transduction
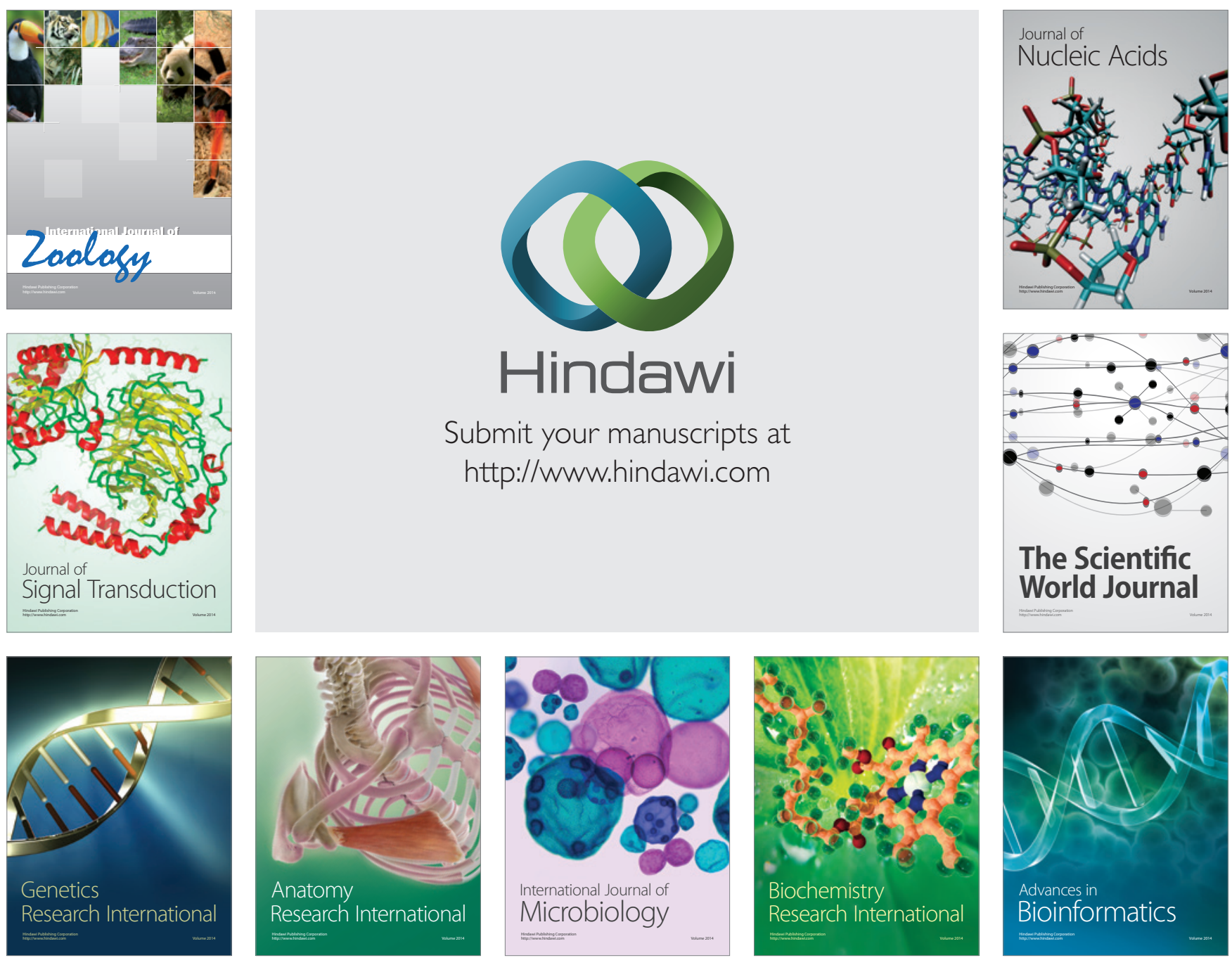

The Scientific World Journal
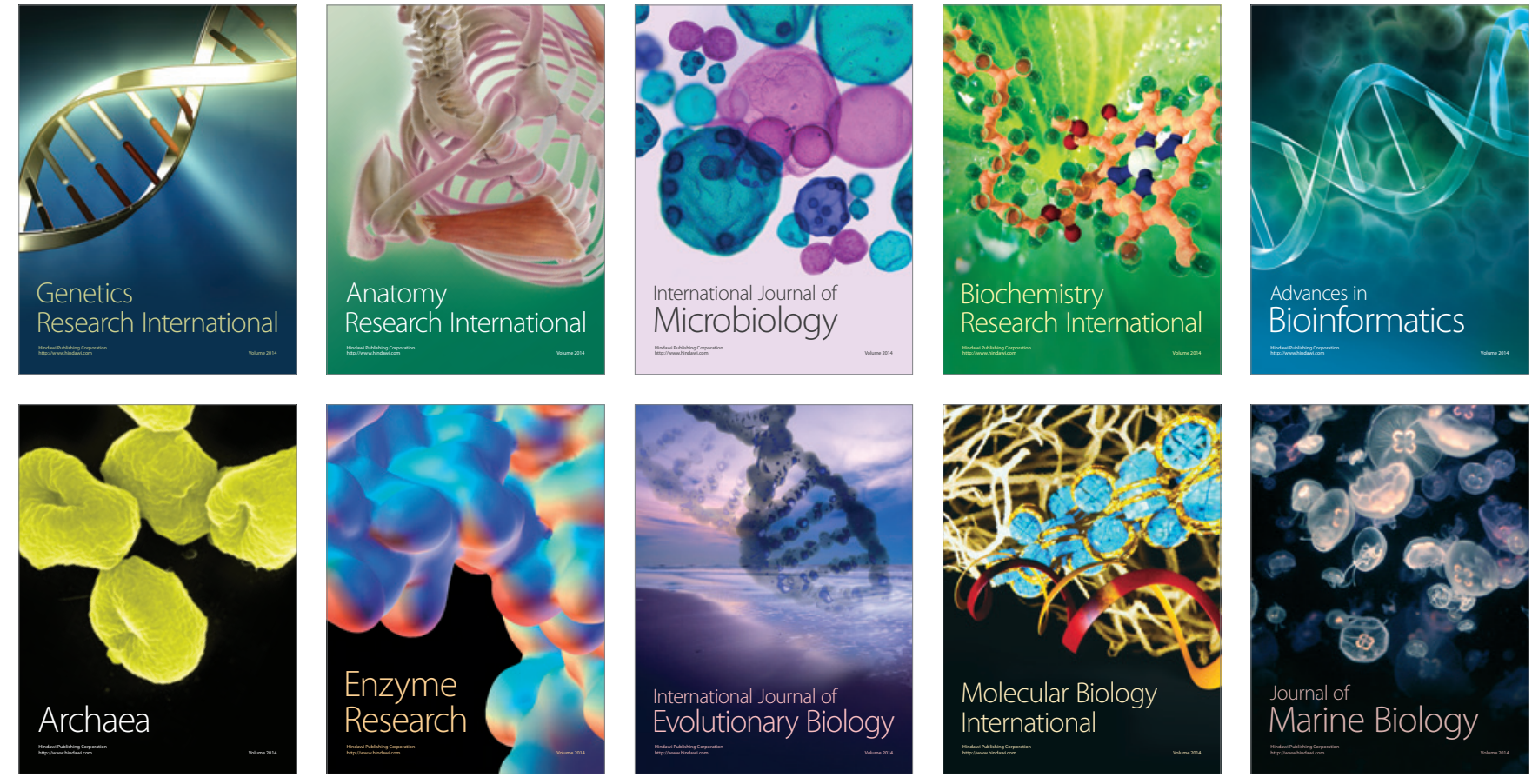